\title{
Hyperopic laser correction
}

\begin{abstract}
Laser in situ keratomileusis (LASIK) of hyperopic eyes is frequently associated with regression and the need of a retreatment. Several studies were studying the potential aetiologies of post-LASIK regression in hyperopia including flap creation, treatment optical zone, blend zone, corneal epithelial hyperplasia and stromal hyperplasia. All these factors contribute to poor postoperative predictability and efficacy. By examining these factors and modifying certain technical steps of the procedure, the refractive surgeon may be able to improve visual outcome and decrease the rate of retreatments.
\end{abstract}

Keywords: LASIK, hyperopia, regression, refractive surgery
Volume 8 Issue I - 2018

Osama Elbassiouny, Ehab Moawad

Department of Ophthalmology, Suez Canal University, Egypt

Correspondence: Osama Elbassiouny, Professor and the Founding Director of Ophthalmology Department, Suez Canal University, Ismailieh, Egypt,

Email Osama.elbassiouny@medsuez.edu

Received: January 28, 2018 | Published: February 06, 2018

\section{Discussion}

There is less predictability of LASIK treatment in hyperopic eyes than in myopic eyes because with hyperopic eyes there is a higher risk of regression and a greater tendency toward losing corrected distance visual acuity (CDVA) ${ }^{1-3}$ Regression is defined as a return divergence of refraction from the targeted refraction toward the original refractive error mostly occurs 3-6 months after refractive surgery. It was a common complication with the previous generation of refractive surgeries, such as radial keratotomy RK) and photo-refractive keratectomy (PRK), but seems to occur less after myopic LASIK. In Hyperopic LASIK technique, applying the peripheral ablation under the corneal flap, a more stable and predictable refractive outcome can be obtained than that achieved by surface based H-PRK because stromal healing and epithelial hyperplasia is inhibited. However we need a large corneal flap of at least $9 \mathrm{~mm}$ in diameter to apply a sufficiently large peripheral ablation zone that can be more difficult in the small hyperopic eye with a relatively flat cornea and also hyperopic treatment zone diameters are often equal or exceed the size of the stromal bed created by most microkeratomes. ${ }^{2}$ Thus, Hyperopic refractive laser correction represents a challenge for most refractive surgeons. ${ }^{3}$ Although LASIK is considered the preferred technique by most refractive surgeons, hyperopic laser correction is challenging, starting from accurate assessment of patient manifest refraction and integration of the cycloplegic refraction in this process followed by accurate centration during the surgery. ${ }^{2-4}$ Basically, the aim of excimer laser ablation hyperopic LASIK correction is to induce steepening in the central optical zone of the cornea; this is obtained by an annular ablation profile, which induces flattening in the corneal periphery; thus, it is considered more difficult and less predictable than the myopic ablation, which induces direct flattening in the central corneal area. $^{5}$

Another issue is that hyperopic ablation time is longer than the equivalent dioptric correction for myopic refraction, which increases the risk of the patient eye movement during surgery and requires an accurate and fast eye-tracking system in order to ensure that you deliver laser ablation in the planned treatment zone; ${ }^{5}$ this problem may be accentuated by the fact that most of the hyperopic patient who are seeking refractive surgery are relatively old already presbyopic and have a problem to fixate to a near target during ablation, which may lead to de-centration of the ablation. This problem is overcome to a great extent by the newer, accurate eye-trackers combined with faster excimer laser systems. ${ }^{10,11}$ Another dilemma is where to locate the ablation profile: the corneal center, the pupil center, or somewhere in between in order to target the true visual axis; the problem is even more complicated in hyperopic patients, as they usually have a wide angle Kappa ${ }^{6}$ Incidence of epithelial in growth is greater in hyperopic LASIK because of the peripheral ablation adjacent to the edge of the flap so the epithelium might gain access under the flap to the deep ablated zone. ${ }^{7}$ An important issue is the greater tendency of regression has been reported after hyperopic LASIK, which may be explained by postoperative loss of accommodative spasm, mechanical instability of corneal biomechanics, and/or irregular epithelial remodeling over the ablation zone. ${ }^{2}$ Postoperative epithelial or sub-epithelial and stromal hyperplasia leading to postoperative corneal steepening has been implicated in the aetiology of postoperative refractive regression. It is not clear yet which layer plays a more prominent role in this postoperative complication. ${ }^{3}$ although the Laser machines are cleared to treat up to $+6.00 \mathrm{D}$, most studies report an upper limit of not more than +4.00 . LASIK for patients with low degrees of hyperopia has shown in several studies greater safety, efficacy, and predictability. However in patients with higher degrees of hyperopia, the outcomes are less predictable, and regression is more common., ${ }^{2,4,5}$ In a 5-year study follow-up period of patients with +0.75 to $+7.00 \mathrm{D}$ of hyperopia, LASIK showed moderately efficacy and predictability for correcting low degrees of hyperopia, but regression occurred throughout the study period. Hyperopic LASIK was effective and safe in correction up to $+3.75 \mathrm{D}$, and other studies reported upper limits of $+5.000^{2,6} \mathrm{In}$ addition to applying new advancements in laser- delivery platforms, several approaches have been justified to improve the outcomes of hyperopic LASIK treatments including nomogram adjustments, larger optical zone, blend zone and the size of the flap, altering the centration of the ablation treatment (i.e., proper estimation of the visual axis), and application of wavefront technology. ${ }^{7-9}$ Zadok et al. ${ }^{10}$ performed hyperopic LASIK on 72 eyes up to $+5.00 \mathrm{D}$ and reported good predictability in correction up to +3.0 diopters with $89 \%$ of eyes within plus or minus $1.0 \mathrm{D}$ of emmetropia but predictability was less at levels more than $+3.0 \mathrm{D}(52 \%$ within plus or minus $1.0 \mathrm{D}$ of emmetropia). Significant regression was reported in both low and moderate hyperopia with $20 \%$ of eyes in the low and $33.3 \%$ in the moderate hyperopic groups needed retreatment. However, Davidorf et al. ${ }^{11}$ reported that while hyperopic correction can be properly achieved with optical zones from 5.0 to $6.0 \mathrm{~mm}$ diameter, there is a tendency towards overcorrection with wider optical zones. In the opinion of 
three of the expert panel, hyperopic LASIK is the best approach for correction of low to moderate hyperopia (to a maximum of $+4.00 .{ }^{12,13}$ Mitomycin C (MMC) has been recently used by refractive surgeons to prophylactically prevent postoperative haze after surface ablation procedures and therapeutically applied in the treatment of preexisting haze. ${ }^{14}$ Drake et al. ${ }^{15}$ assessed the role of mitomycin C in Hyperopic femto-LASIK. They reported that hyperopic eyes treated with MMC showed less regression 3 months after surgery and had better visual outcome and a smaller residual sphere. Furthermore, the application of MMC of concentration of $0.02 \%$ the stromal bed after laser ablation for 5 seconds during Femtosecond surgery seemed to have a better predictability (spherical equivalent within $\pm 0.50 \mathrm{D}$ of targeted refraction at 3 months) than when MMC was not applied.

\section{Conclusion}

Refractive surgeons should take into account some considerations and few modifications when performing hyperopic laser correction in order to improve the visual outcome and achieve greater predictability and efficacy and thus decreasing the rate of regression and the need for retreatment. These measures include defining upper limit of hyperopic correction of not more than $+3,0$ diopters, proper ablation profile with fast laser machines and high speed eye-trackers, proper flap creation (better femtolaser assisted than microkeratomes) large optical one of (6-7 $\mathrm{mm})$ and proper blend zone.

\section{Acknowledgments}

None.

\section{Funding}

None.

\section{Conflicts of interest}

None.

\section{References}

1. Mohamed TA, Hoffman RS, Fine IH, et al. Post-laser assisted in situ keratomileusis epithelial ingrowth and its relation to pretreatment refractive error. Cornea. 2011;30(5):550-552.

2. Alió JL, El Aswad A, Vega-Estrada A, et al. Laser in situ keratomileusis for high hyperopia ( $>5.0$ diopters) using optimized aspheric profiles: efficacy and safety. J Cataract Refract Surg. 2013;39(4):519-527.
3. Helena MC, Baerveldt F, Kim WJ, et al. Keratocyte apoptosis after corneal surgery. Invest Ophthalmol Vis Sci. 1998;39(2):276-283.

4. Jaycock PD, O'Brart DP, Rajan MS, et al. 5-year follow-up of LASIK for hyperopia. Ophthalmology. 2005;112(2):191-199.

5. Tanzer DJ, Brunstetter T, Zeber R, et al. Laser in situ keratomileusis in United States Naval aviators. J Cataract Refract Surg. 2013;39(7):1047-1058.

6. Llovet F, Galal A, Benitez-del-Castillo JM, et al. One-year results of excimer laser in situ keratomileusis for hy- peropia. $J$ Cataract Refract Surg. 2009;35(7):1156-1165.

7. Zaldivar R, Oscherow S, Bains HS. Five techniques for improving outcomes of hyperopic LASIK. J Refract Surg. 2005;21(5 Suppl):S628S632.

8. Durrie DS, Smith RT, Waring GO, et al. Comparing conventional and wavefront- optimized LASIK for the treatment of hyperopia. $J$ Refract Surg. 2010;26(5):356-363.

9. Mosquera SA, Gmbh S, Kg C. Hyperopic laser in situ keratomileusis: Comparison of femtosecond laser and mechanical microkeratome flap creation Hyperopic laser in situ keratomileusis: Comparison of femtosecond laser and mechanical microkeratome flap creation. 2016.

10. Zadok D, Maskaleris G, Montes M, et al. Hyperopic laser in situ keratomileusis with the Nidek EC-5000 excimer laser. Ophthalmology. 2000;107:1132-1137.

11. Davidorf JM, Eghbali F, Onclinx T, et al. Effect of varying the optical zone diameter on the results of hyperopic laser in situ keratomileusis. Ophthalmology. 2001;108:1261-1265.

12. Schnitzler EM, Baumeister M, Kohnen T. Scotopic measurement of normal pupils: Colvard versus Video Vision Analyzer infrared pupillometer. $J$ Cataract Refract Surg. 2000;26(6):859-866.

13. El-naggar MT, Hovaghimian DG. Assessment of refractive outcome of femtosecond-assisted LASIK for hyperopia correction. Electron Physician. 2017;9(3):3958-3965.

14. Santhiago MR, Netto MV, Wilson SE. Mitomycin C: Biological Effects and Use in Refractive Surgery. Cornea. 2012;31(3):311-321.

15. P Drake, J Paz Moreno-Arrones, M Garcia Gonzalez, et al. Femtosecondassisted LASIK with or without intraoperative Mitomycin C (MMC) to correct hyperopia. ESCRS. 2014. 